Pacific Journal of Mathematics

BOUNDARY BEHAVIOR OF LIMITS OF DISCRETE SERIES
REPRESENTATIONS OF REAL RANK ONE SEMISIMPLE
GROUP BRIAN EVAN BLANK 


\title{
BOUNDARY BEHAVIOR OF LIMITS OF DISCRETE SERIES REPRESENTATIONS OF REAL RANK ONE SEMISIMPLE GROUPS
}

\author{
BRIAN BLANK
}

\begin{abstract}
The decomposition of the reducible unitary principal series of a connected semisimple Lie group having real rank one and a simply connected complexification is exhibited on a global analytic level in such a way that it is seen to correspond to a phenomenon in classical Fourier analysis. this is done by embedding limits of discrete series representations via a group equivariant passage to boundary values analogous to the classical Hardy space inclusion used by Bargmann in the case of $\operatorname{SL}(2, R)$. The boundary value map is shown to be a factor of the projection operator given by the Knapp-Stein intertwining operator. From a representation theoretic view, while these decompositions are already known, the method of computing the leading term of the asymptotic expansion of matrix coefficients is new and does not require a $K$-finiteness assumption.
\end{abstract}

1. Introduction and preliminaries. The decomposition of representations in the unitary principal series of a connected semisimple Lie group $G$ having real rank one and a simply connected complexification is well understood [10], [11]. In particular, Knapp and Wallach having used Szegö kernels to decompose all reducible unitary principal series representations as sums of limits of discrete series representations [11, §12]. In this paper we exhibit these reducibility results on a global analytic level by explicitly embedding limits of discrete series representations in the reducible principal series. This is achieved by realizing the representations in question in suitable function spaces and providing a group equivariant passage to boundary values analogous to the Hardy space inclusion of $H^{2}(\mathbf{R})$ in $L^{2}(\mathbf{R})$ that was used by Bargmann in the case $G=\operatorname{SL}(2, \mathbf{R})[1]$ and Knapp and Okamoto [9] more generally in the case of limits of holomorphic discrete series.

Throughout this paper we assume that $G$ satisfies the properties listed above. Furthermore, from the point of view of exhibiting reducibility results, there is no loss of generality in assuming that $G$ has a compact Cartan subgroup $T \subseteq K$ where $K$ is a maximal compact subgroup of $G$ corresponding to a Cartan involution $\theta[10$, p. 543-544]. Then $G$ has discrete series $\mathscr{E}^{2}(G)$ [5]. To each nonsingular integral form $\Lambda$ on the Lie algebra $\mathfrak{t}$ of $T$, Harish-Chandra associates an invariant eigendistribution 
$\Theta_{\Lambda}[4$, Theorem 2] and proves the existence of a discrete series representation $\left(\pi_{\Lambda}, H^{\Lambda}\right)$ with character $\Theta_{\Lambda}[5]$ and that these representations exhaust $\mathscr{E}^{2}(G)$; we call $\Lambda$ a Harish-Chandra parameter.

Let $\mathfrak{g}$ and $\mathfrak{f}$ denote the Lie algebras of $G$ and $K$, and let $\Delta$ (respectively $\Delta_{k}, \Delta_{n}$ ) denote the roots (respectively compact roots, noncompact roots) of $\left(g^{\mathbf{C}}, t^{\mathbf{C}}\right)$. Normalize root vectors $E_{\alpha}(\alpha \in \Delta)$ according to $[6,155-156]$. If $\Lambda$ is a Harish-Chandra parameter we order $\Delta$ so that $\Lambda$ is $\Delta^{+}$-dominant; $\Delta^{+}$is thereby uniquely determined. If instead the integral parameter $\Lambda$ is singular, but not orthogonal to any compact root, it is easy to see that there is a noncompact root $\alpha$ for which $\{ \pm \alpha\}$ is precisely the set of roots orthogonal to $\Lambda[11$, Lemma 12.5]. For such a parameter, called here a limit Harish-Chandra parameter, there are two possible choices of positive roots $\Delta^{+}$for which $\Lambda$ is $\Delta^{+}$-dominant. Whichever the choice, the unique positive root orthogonal to $\Lambda$ is noncompact and simple [11, Lemma 12.5].

Let $\Lambda$ be either a Harish-Chandra parameter or a limit HarishChandra parameter. Order $\Delta$ so that $\Lambda$ is $\Delta^{+}$-dominant and put $\delta$ $=\frac{1}{2} \sum_{\alpha \in \Delta^{+}} \alpha, \delta_{k}=\frac{1}{2} \sum_{\alpha \in \Delta_{k}^{+}} \alpha$, and $\delta_{n}=\delta-\delta_{k}$. Let $\alpha_{0}$ be any simple noncompact root if $\Lambda$ is nonsingular and the unique positive root orthogonal to $\Lambda$ (hence also simple noncompact) if $\Lambda$ is singular. Then $\alpha_{0}$ is a fundamental sequence of positive noncompact roots in the sense of [11, §4] and $\alpha_{0}$ determines an Iwasawa decomposition $G=A N K$ with the Lie algebra $a$ of $A$ given by $\mathfrak{a}=\mathbf{R} \cdot\left(E_{\alpha_{0}}+E_{-\alpha_{0}}\right)$ and $E_{\alpha_{0}}+E_{-\alpha_{0}}$ in the positive chamber of $a$. Observe that if $\Lambda$ is singular, the Iwasawa decomposition does not depend on which of the two possible systems of positive roots $\Delta^{+}$that is used. Let $M$ (respectively $M^{\prime}$ ) denote the centralizer (respectively normalizer) of $A$ in $K$ and denote by $P$ the minimal parabolic subgroup $M A N$ of $G$. Let $\lambda=\Lambda-\delta_{k}+\delta_{n}$ be the Blattner parameter corresponding to $\left(\Lambda, \Delta^{+}\right)$. Thus, when $\Lambda$ is nonsingular, $\lambda=\lambda(\Lambda)$ is the lowest $K$-type in $\pi_{\Lambda}$. Even when $\Lambda$ is singular, $\lambda$ is integral and $\Delta_{k}^{+}$-dominant [11, p. 198]; the Blattner parameter $\lambda^{\prime}$ corresponding to $\left(\Lambda, \Delta^{+\prime}\right)$, where $\Delta^{+^{\prime}}=\left(\Delta^{+}-\left\{\alpha_{0}\right\}\right) \cup\left\{-\alpha_{0}\right\}$ is the other possible positive root system, is given by $\lambda^{\prime}=\lambda-\alpha_{0}$. For $\mu$ integral and $\Delta_{k}^{+}$-dominant let $\left(\tau_{\mu}, V_{\mu}\right)$ denote an irreducible unitary representation of $K$.

A convenient realization of the discrete series representation $\pi_{\Lambda}(\Lambda$ nonsingular) is one the space of square integrable functions in

$$
\begin{aligned}
& C^{\infty}\left(G, \tau_{\Lambda}\right) \\
& \quad=\left\{F \in C^{\infty}\left(G, V_{\lambda}\right) \mid F(k g)=\tau_{\lambda}(k) F(g), k \in K, g \in G\right\}
\end{aligned}
$$


that are annihiliated by a certain first order elliptic differential operator $\mathscr{D}_{\lambda}[13,14](\lambda=\lambda(\Lambda))$.

For $\nu \in \operatorname{Hom}_{\mathbf{R}}(\mathfrak{a}, \mathbf{C})=\mathfrak{a}_{\mathbf{C}}^{\prime}$ and $(\sigma, H)$ a irreducible unitary representation of $M$, let $U(\sigma: \nu)$ denote the nonunitary principal series representation realized in the compact picture on $L^{2}(K, \sigma)$ (cf. §2). In [11] Knapp and Wallach associate to the parameter $\Lambda$ (and the ordering $\Delta^{+}$if $\Lambda$ is nonsingular) an irreducible unitary representation $\left(\sigma_{\lambda}, H_{\lambda}\right)$ of $M$ with highest weight $\lambda$ and $H_{\lambda} \subset V_{\lambda}\left(\lambda=\lambda\left(\Lambda, \Delta^{+}\right)\right)$, a parameter $\nu(\lambda)$ in $\mathfrak{a}_{\mathbf{C}}^{\prime}$, and an integral formula $S_{\lambda}$ defined on the dense subspace

$$
\begin{aligned}
C^{\infty}( & \left.K, \sigma_{\lambda}\right) \\
& =\left\{f \in C^{\infty}\left(K, H_{\lambda}\right) \mid f(m k)=\sigma_{\lambda}(m) f(k), m \in M, k \in K\right\}
\end{aligned}
$$

of $L^{2}\left(K, \sigma_{\lambda}\right)$ by

$$
S_{\lambda} f(x)=\int_{K} \tau_{\lambda}(k)^{-1} f(k x) d k \quad(x \in G) .
$$

The dependence on $v(\lambda)$ is incorporated into the extension of $f$ to $G$ required for formula (1.3) (cf. §2). The point is that $S_{\lambda}$ carries $C^{\infty}\left(K, \sigma_{\lambda}\right)$ $G$-equivariantly into the kernel of $\mathscr{D}_{\lambda}$ in $C^{\infty}\left(G, \tau_{\lambda}\right)$ and thus provides a quotient map of $U\left(\sigma_{\lambda}: \nu(\lambda)\right)$ onto $\pi_{\Lambda}$ when $\Lambda$ is nonsingular. When $\Lambda$ is singular, the two Blattner parameters $\lambda$ and $\lambda^{\prime}$ give rise to equivalent $M$-types $\sigma_{\lambda}$ and $\sigma_{\lambda^{\prime}}$ and the formulas for both $\nu(\lambda)$ and $\nu\left(\lambda^{\prime}\right)$ reduce to $\rho$ (cf. §2). The unitary principal series representations $U\left(\sigma_{\lambda}: \rho\right)$ and $U\left(\sigma_{\lambda^{\prime}}: \rho\right)$ are therefore equivalent. Nevertheless, their images under the Szegö maps $S_{\lambda}$ and $S_{\lambda^{\prime}}$ respectively have lowest $K$-types $\lambda$ and $\lambda^{\prime}=\lambda-\alpha_{0}$ respectively and so are independent limits of discrete series representations. Knapp and Wallach showed that the unitary principal series representation $U\left(\sigma_{\lambda}: \rho\right)$ is infinitesimally equivalent with the direct sum of the $K$-finite images of $U\left(\sigma_{\lambda}: \rho\right)$ and $U\left(\sigma_{\lambda^{\prime}}: \rho\right)$ under $S_{\lambda}$ and $S_{\lambda^{\prime}}$ respectively [11, Theorem 12.6]. The completeness result that all reducibility of the unitary principal series may be so accounted for is Theorem 12.7 of [11].

In this paper we will establish this decomposition in a global analytic fashion by means of a boundary value embedding $\mathscr{L}$ carried out in $\S 3$. The point is that, although $\lim _{a \rightarrow \infty} S_{\lambda} f(a)=0$ for $f$ in $C^{\infty}\left(K, \sigma_{\lambda}\right)$, we can write $S_{\lambda} f(a)=c(f) e^{-\rho \log a}+$ lower order terms, with $c(f) \neq 0$ in general. The boundary map is then defined by the constant term in the expansion of $e^{\rho \log a} S_{\lambda} f(a)$ after projecting by $E_{\lambda}$ from $V_{\lambda}$ onto $H_{\lambda}$ :

$$
\mathscr{L}\left(S_{\lambda} f\right)(k)=E_{\lambda} c\left(U\left(\sigma_{\lambda}: \rho: w^{-1} k\right) f\right)
$$

where $w$ is a certain representative of the nontrivial Weyl group element. The bulk of $\S 3$ is devoted to establishing the finite, generally non-zero 
limit in (1.4). The main tools in this analysis were developed in [10], which we quote frequently. Particularly important for our purposes are the mean value property [10, Proposition 20]

$$
\int_{c<|v|<d} \frac{\sigma_{\lambda}(v w)^{-1}}{|v|} d v=0
$$

and another result of Knapp-Stein which we include here as Lemma 3.1. Some consequences of the proof of this lemma, such as Proposition 3.3 and 3.4, may be of independent interest. The final limit result needed to define $\mathscr{L}$ is given in Theorem 3.14. In Theorem 3.16 it is shown that $\mathscr{L}$ maps the limit of discrete series represenation with lowest $K$-type $\lambda$ $G$-equivariantly into $U\left(\sigma_{\lambda}: \rho\right)$.

Embedding theorems for limits of discrete series for the classical real rank one groups were given in [12]. In addition to the greater generality of the present paper, the results given here may be of interest through their relationship with the Knapp-Stein intertwining operators. These were given in [10] where it is shown $[\mathbf{1 0}, \mathrm{p} .517]$ that in the noncompact picture $\mathscr{U}\left(\sigma_{\lambda}: \rho\right)$ the intertwining operators consist of linear combinations of the identity and the convolution operator with kernel $|v|^{-1} \sigma_{\lambda}(v w)^{-1}$. We show in Theorem 3.16 that the composition

$$
\mathscr{U}\left(\sigma_{\lambda}: \rho\right) \stackrel{W^{-1}}{\rightarrow} U\left(\sigma_{\lambda}: \rho\right) \stackrel{S_{\lambda}}{\rightarrow} \quad \begin{gathered}
\text { Limit of } \\
\text { Discrete Series }
\end{gathered} \stackrel{\mathscr{L}}{\rightarrow} U\left(\sigma_{\lambda}: \rho\right) \stackrel{W}{\rightarrow} \mathscr{U}\left(\sigma_{\lambda}: \rho\right)
$$

(cf. $\$ 2$ for the definition of $W$ ) is indeed of the type described.

Some of these results were announced in [2]. It is a pleasure to thank Professors A. W. Knapp and N. R. Wallach for their valuable suggestions.

2. The Szegö integral. Let $\Lambda$ be a limit Harish-Chandra parameter. Order $\Delta$ so that $\Lambda$ is $\Delta^{+}$-dominant and let $\alpha_{0}, A$, and $a$ be as defined in $\S 1$. Let $\Phi$ denote the restricted roots of $\mathfrak{g}$ with respect to $\mathfrak{a}$; for $\gamma \in \Phi$ let $\mathrm{g}^{\gamma}$ denote the corresponding restricted root space and set $\gamma \in \Phi^{+}$if $\gamma\left(E_{\alpha_{0}}+E_{-\alpha_{0}}\right)>0$. Our assumption that $G$ has real rank one results in $\Phi^{+}$having the form $\Phi^{+}=\{\alpha\}$ or $\Phi^{+}=\{\alpha, 2 \alpha\}$. In our notation $\alpha$ will denote the smallest positive root. Let $p=\operatorname{dim}_{\mathbf{R}} \mathrm{g}^{\alpha}, q=\operatorname{dim}_{\mathbf{R}} \mathrm{g}^{2 \alpha}$ where $\mathrm{g}^{2 \alpha}=(0)$ if $2 \alpha \notin \Phi$, and let $\rho$ denote half the sum of the positive restricted roots with multiplicity so that

$$
\rho=\frac{1}{2}(p+2 q) \alpha .
$$

Let $\mathfrak{n}=\sum_{\beta \in \Phi} \oplus \mathfrak{g}^{\beta}$ and let $N$ and $\bar{N}$ denote the analytic subgroups of $G$ corresponding to $\mathfrak{n}$ and $\theta \mathfrak{n}$. 
Since $\operatorname{dim}_{\mathbf{R}} \mathfrak{a}=1$, the Weyl group $\mathfrak{w}=M^{\prime} / M$ has order two. Let $M^{\prime}$ act in each equivalence class $[\sigma]$ in $\hat{M}$, the set of equivalence classes of irreducible unitary representations of $M$, by

$$
w \sigma(m)=\sigma\left(w^{-1} m w\right) \quad\left(w \in M^{\prime} ; m \in M:[\sigma] \in \hat{M}\right) .
$$

By [8] we can choose a representation $w$ of the nontrivial Weyl group element that centralizes $M$ so that

$$
w \sigma=\sigma .
$$

We denote the factors of an element $g$ in the Iwasawa decomposition $G=A N K$ by

$$
g=\exp H(g) \cdot n \kappa(g) \quad(H(g) \in a, \kappa(g) \in K)
$$

and write $\log a$ for $H(a)$ when $a \in A$. Every element $g$ not in the lower dimensional set $P w$ where $P=M A N$ also has a unique Gelfand-Naimark decomposition

$$
g=m(g) a(g) n \bar{n}(g)
$$

with factors in $M, A, N$, and $\bar{N}$ respectively. By means of this decomposition we extend representations $\sigma$ of $M$ and characters $\chi$ of $A$ to functions defined almost everywhere on $G$ with respect to Haar measure:

$$
\sigma(\operatorname{man} \bar{n})=\sigma(m), \quad \chi(\operatorname{man} \bar{n})=\chi(a)
$$

where we adopt without further reference the lower case convention for group elements with the exception that $v$ will always denote an element of $\bar{N}$. The Bruhat decomposition shows that for each $g$ in $G$ there is at most one $v$ in $\bar{N}$ for which $\bar{n}(v g)$ is not defined. If $\bar{N}_{g}$ is this exceptional set, then $\bar{N}_{w}=\bar{N}-\{1\}$.

Let $\lambda$ be the Blattner parameter associated to $\left(\Lambda, \Delta^{+}\right)$as described in $\S 1$ and let $\left(\tau_{\lambda}, V_{\lambda}\right)$ denote a $K$-type with highest weight $\lambda$. Let $\phi_{\lambda}$ be a highest weight vector of $\tau_{\lambda}$ of length one, let $H_{\lambda}$ be the $M$-cyclic subspace of $V_{\lambda}$ generated by $\phi_{\lambda}$, and let $\sigma_{\lambda}$ be the representation of $M$ given by $\tau_{\lambda}$ operating on $H_{\lambda}$. The proof of Proposition 5.5 of [11] and Lemma 12.3 of [11] show that $\left(\sigma_{\lambda}, H_{\lambda}\right)$ is an irreducible representation of $M$.

We recall from [3] that

$$
\int_{\bar{N}} e^{(1+z) \rho H(v)} d v<\infty \quad \text { if } \operatorname{Re} z>0
$$

where $d v$ is unimodular Haar measure on $\bar{N}$. We normalize Haar measures on $M, K$, and $\bar{N}$ so that

$$
\int_{M} d m=\int_{K} d k=\int_{\bar{N}} e^{2 \rho H(v)} d v=1
$$


We arrange parameters so that induction of $\sigma_{\lambda} \otimes e^{\rho} \otimes 1$ from $M A N$ to $G$ gives rise to a unitary representation. In the compact picture of this unitary principal series $U=U\left(\sigma_{\lambda}: \rho\right)$ the representation space is the closed subspace $L^{2}\left(K, \sigma_{\lambda}\right)$ of $L^{2}\left(K, H_{\lambda}\right)$ consisting of functions $f$ such that for every $m$

$$
f(m k)=\sigma_{\lambda}(m) f(k)
$$

$d k$-almost everywhere in $K$. The action of $G$ on $L^{k}\left(K, \sigma_{\lambda}\right)$ is

$$
U(g) f(k)=e^{\rho H(k g)} f(\kappa(k g)) .
$$

We let $C^{\infty}\left(K, \sigma_{\lambda}\right)$ denote the space of smooth functions in $L^{2}\left(K, \sigma_{\lambda}\right)$. Then $C^{\infty}\left(K, \sigma_{\lambda}\right)$ is dense and is the space of $C^{\infty}$-vectors for $U$. If $l$ belongs to $K$, it will be convenient to denote its action on $f$ under $U$ by 'f .

In the unitarily equivalent noncompact picture $\mathscr{U}$ of $U$, the Hilbert space is $L^{2}\left(\bar{N}, H_{\lambda}\right)$ and the group action is given by

$$
\mathscr{U}(g) F(v)=e^{\rho \log v g} \sigma(v g) F(\bar{n}(v g)) .
$$

The intertwining operator $W$ between these two pictures is

$$
W f(v)=e^{\rho H(v)} f(\kappa(v)) \quad\left(v \in \bar{N} ; f \in L^{2}\left(K, \sigma_{\lambda}\right)\right) .
$$

For $f$ in $C^{\infty}\left(K, \sigma_{\lambda}\right)$ Knapp and Wallach define the Szegö map $S_{\lambda}$ with parameter $\lambda$ by

$$
S_{\lambda} f(x)=\int_{K} e^{\rho H\left(l x^{-1}\right)} \tau_{\lambda}\left(\kappa\left(l x^{-1}\right)\right)^{-1} f(l) d l \quad(x \in G) .
$$

Extending $f$ in $C^{\infty}\left(K, \sigma_{\lambda}\right)$ to $G$ by $\tilde{f}(g)=e^{\rho H(g)} f(\kappa(g))$ so that $\tilde{f}(\operatorname{man} x)$ $=e^{\rho \log a} \sigma_{\lambda}(m) \tilde{f}(x)$ and $\tilde{f}$ is in the induced picture of $U$, we have [11, p. $178]$

$$
S_{\lambda} \tilde{f}(x)=\int_{K} \tau_{\lambda}(k)^{-1} \tilde{f}(k x) d k \quad(x \in G),
$$

exhibiting the $G$-equivariance of the Szegö map into the space $C^{\infty}\left(G, \tau_{\lambda}\right)$. It is shown in [11] that the image of $C^{\infty}\left(K, \sigma_{\lambda}\right)$ under $S_{\lambda}$ is in the kernel of $\mathscr{D}_{\lambda}$ in $C^{\infty}\left(G, \tau_{\lambda}\right)$ and that infinitesimally the $K$-finite image of $S_{\lambda}$ is a direct summand of $U\left(\sigma_{\lambda}: \rho\right)$.

We will need another integral formula for the operator $S_{\lambda}$, one that will be of use in conjunction with the noncompact picture $\mathscr{U}\left(\sigma_{\lambda}: \rho\right)$.

LEMMA 2.1. Let $f$ belong to $C^{\infty}\left(K, \sigma_{\lambda}\right)$ and let $S_{\lambda}$ be defined by (2.12) or the equivalent formula (2.13). Then

$$
S_{\lambda} f(a)=\int_{\bar{N}} e^{\rho H(v a)} \tau_{\lambda}(\kappa(v a) w)^{-1}\left(W^{w} f\right)(v) d v .
$$


Proof. We use the integral formula

$$
\int_{K} \varphi(k) d k=\int_{\bar{N}} \int_{M} \varphi(m \kappa(v)) e^{2 \rho H(v)} d m d v
$$

of Harish-Chandra [3, p. 287]. Thus, since $w a^{-1} w^{-1}=a, H(m \kappa(v) a)=$ $-H(v)+H(v a)$, and $\kappa(m \kappa(v) a)=m \kappa(v a)$, we have

$$
\begin{aligned}
S_{\lambda} f(a) & =\int_{K} e^{\rho H\left(l a^{-1}\right)} \tau_{\lambda}\left(\kappa\left(l a^{-1}\right)\right)^{-1} f(l) d l \\
& =\int_{K} e^{\rho H(l a)} \tau_{\lambda}(\kappa(l a) w)^{-1} f(l w) d l \\
& =\int_{\bar{N}} \int_{M} e^{\rho H(m \kappa(v) a)} \tau_{\lambda}(\kappa(m \kappa(v) a) w)^{-1}{ }^{w} f(m \kappa(v)) e^{2 \rho H(v)} d m d v \\
& =\int_{\bar{N}} \int_{M} e^{\rho H(v)} e^{\rho H(v a)} \tau_{\lambda}(\kappa(v a) w)^{-1} \tau_{\lambda}(m)^{-1} \sigma_{\lambda}(m)^{w} f(\kappa(v)) d m d v \\
& =\int_{\bar{N}} e^{\rho H(v a)} \tau_{\lambda}(\kappa(v a) w)^{-1} e^{\rho H(v) w} f(\kappa(v)) d v .
\end{aligned}
$$

In view of the $G$-equivariance of $S_{\lambda}$ this formula can be used globally on $G$ via the Cartan decomposition:

$$
S_{\lambda}(f: k a k)=\tau_{\lambda}(k) S_{\lambda}\left({ }^{k} f: a\right) .
$$

Let $\mathscr{S}_{\lambda}: L^{2}\left(\bar{N}, H_{\lambda}\right) \rightarrow C^{\infty}\left(A, V_{\lambda}\right)$ be defined by

$$
\mathscr{S}_{\lambda} F(a)=e^{\rho \log a} \int_{\bar{N}} e^{\rho H(v a)} \tau_{\lambda}(\kappa(v a) w)^{-1} F(v) d v .
$$

By abuse of notation we define $\mathscr{S}_{\lambda}$ on $C^{\infty}\left(K, \sigma_{\lambda}\right)$ by

$$
\mathscr{S}_{\lambda} f(a)=e^{\rho \log a} S_{\lambda} f(a)
$$

then we have by Lemma 2.1

$$
\mathscr{S}_{\lambda} f=\mathscr{S}_{\lambda}\left(W^{w} f\right)
$$

3. Boundary values of Szegö integrals. The group $A$ acts on $\bar{N}$ by the dilations $\delta_{a}$ where

$$
\delta_{a} v=a^{-1} v a \quad(v \in \bar{N})
$$

with change of variables given by

$$
d\left(\delta_{a} v\right)=e^{2 \rho \log a} d v .
$$


The homogeneous norm $|v|$ on $\bar{N}[\mathbf{1 0}, \mathrm{p} .512]$ given by

$$
|v|=e^{-\rho \log (v w)} \quad\left(v \in \bar{N}_{w}=\bar{N}-\{1\}\right)
$$

is $\alpha$-homogeneous of degree $p+2 q$ and invariant under conjugation by $M$, i.e.,

$$
\left|\delta_{a} v\right|=e^{2 \rho \log a}|v| \text { and }\left|\mathrm{mum}^{-1}\right|=|v| \quad(v \neq 1) .
$$

The function $v \rightarrow \sigma_{\lambda}(v w)$ is of class $C^{\infty}$ away from $v=1$ and has the homogeneity property

$$
\sigma_{\lambda}\left(\delta_{a} v \cdot w\right)=\sigma_{\lambda}(v w) .
$$

These facts may be found in [10, §6 and §8]. The following lemma may also be found in [10] but we provide an outline of its proof because we will need several consequences of the proof not found in [10].

LEMMA 3.1. ([10, Lemma 29].) The map $v \rightarrow|v|^{2}$ is a polynomial on $\bar{N}$ that is $\alpha$-homogeneous of degree less than $2(p+2 q)$ such that

$$
e^{-2 \rho H(v)}=1+P_{1}(v)+\cdots+P_{s}(v)+|v|^{2} .
$$

Consequently $e^{2 \rho H(v)} \leq 1$ and

$$
e^{2 \rho H(v)} \leq \frac{1}{|v|^{2}} \quad(v \neq 1)
$$

Proof (sketch). Let $\pi$ be a finite dimensional irreducible representation with a-weights $\rho=\mu_{0}, \mu_{1}, \ldots, \mu_{s+1}$ of which $\rho$ is the highest and such that the compact real form $\mathfrak{f} \oplus \mathfrak{i p}$ of $\mathrm{g}^{\mathbf{C}}$ acts by skew-Hermitian transformations. If $\phi_{\rho}$ is a highest a-weight vector of length one then $\left\|\pi(g)^{-1} \phi_{\rho}\right\|^{2}=e^{-2 \rho H(g)}$. Let $E_{\mu_{t}}$ denote the orthogonal projection onto the weight space belonging to $\mu_{i}$ and put $P_{j}(g)=\left\|E_{\mu_{j}} \pi(g)^{-1} \phi_{\rho}\right\|^{2}$. Then

$$
e^{-2 \rho H(g)}=P_{0}(g)+\cdots+P_{s+1}(g) \quad(g \in G) .
$$

Routine computation shows that when gw belongs to $M A N \bar{N}$

$$
\left\|E_{w \rho} \pi(g)^{-1} \phi_{\rho}\right\|^{2}=e^{-\rho \log a(g w)}
$$

and in particular when $v \neq 1$

$$
\left\|E_{w \rho} \pi(v)^{-1} \phi_{\rho}\right\|^{2}=e^{-2 \rho \log (v w)} .
$$

Since $P_{s+1}(v)=e^{-2 \rho \log (v w)}=|v|^{2}$, all statements follow from (3.8) and (3.10). 
We define the kernel $K(v: a)$ on $\bar{N} \times A$ by

$$
K(v: a)=e^{2 \rho \log a} e^{\rho H\left(\delta_{a} v\right)} .
$$

Then for $f$ in $C^{\infty}\left(K, \sigma_{\lambda}\right)$

$$
\mathscr{S}_{\lambda} f(a)=\int_{\bar{N}} K(v: a) \tau_{\lambda}\left(\kappa\left(\delta_{a} v\right) w\right)^{-1} W^{w} f(v) d v .
$$

The notation $a \rightarrow \infty$ will signify $a=\exp t\left(E_{\alpha_{0}}+E_{-\alpha_{0}}\right)$ with $t \rightarrow \infty$.

LEMMA 3.2. For $v$ in $\bar{N}$ different from 1 we have

$$
\begin{aligned}
K(v: a) & \leq \frac{1}{|v|}, \\
\lim _{a \rightarrow \infty} K(v: a) & =\frac{1}{|v|}, \quad \text { and }
\end{aligned}
$$

$$
K(v: a) d v=K\left(\delta_{a} v\right) d\left(\delta_{a} v\right) \quad \text { where } K(v)=K(v: 1) .
$$

Proof. Statement (3.12) follows immediately from (3.7) and (3.4) as does (3.14) from (3.2). Statement (3.13) is a simple consequence of (3.6).

Proposition 3.3. The map $\mu: g \rightarrow e^{-2 \rho \log a(g w)}$ which is defined on $M A N \bar{N} w$ can be continuously extended to $G$ by putting $\mu($ man $)=0$.

Proof. By (3.9), $\mu(g)=\left\|E_{w \rho} \pi(g)^{-1} \phi_{\rho}\right\|^{2}$ when $g$ belongs to $M A N \bar{N} w$. Since $M$ preserves the highest a-weight space of $\pi, A$ acts by scalars, and $N$ acts trivially, $\| E_{w \rho} \pi(\text { man })^{-1} \phi_{\rho} \|^{2}=0$, the result follows because $G=$ $P \cup P \bar{N} w$ (essentially the Bruhat decomposition of $G$ ).

The significance of Proposition 3.3 is that $\left.\mu\right|_{K}$ is zero precisely on $M$ and so provides a means of testing when an element of $K$ belongs to $M$.

Proposition 3.4. For $v$ in $\bar{N}$ different from $1, \lim _{a \rightarrow \infty} \kappa\left(\delta_{a} v\right) w=$ $m(v w)$.

Proof. For every $a$ in $A$ and $v$ in $\bar{N}$, writing $\delta_{a} v$ as $e^{H\left(\delta_{a} v\right)} n \kappa\left(\delta_{a} v\right)$ we get $\kappa\left(\delta_{a} v\right) w^{2}=w^{2} e^{-H\left(\delta_{a} v\right)} n^{\prime} v^{\prime}$ belongs to $M A N \bar{N}$, since $w^{2}$ is in $M$, and so, in particular,

$$
\mu\left(\kappa\left(\delta_{a} v\right) w\right)=e^{-2 \rho \log a\left(\kappa\left(\delta_{a} v\right) w^{2}\right)}=e^{2 \rho H\left(\delta_{a} v\right)} .
$$

Thus, for $v \neq 1$,

$$
\lim _{a \rightarrow \infty} \mu\left(\kappa\left(\delta_{a} v\right) w\right)=\lim _{a \rightarrow \infty} e^{-2 \rho \log a} K(v: a)=0
$$


by (3.12) and so $\kappa\left(\delta_{a} v\right) w$ tends to $M$ as $a \rightarrow \infty$. Since the GelfandNaimark decomposition is continuous on $M A N \bar{N}$, the $A, N$, and $\bar{N}$ components of $\kappa\left(\delta_{a} v\right) w$ for $v \neq 1$ each converge to 1 as $a \rightarrow \infty$. The result follows since $m\left(\kappa\left(\delta_{a} v\right) w\right)=m\left(\delta_{a} v w\right)=m(v w) \quad[\mathbf{1 0}$, formula (6.12)].

Some remarks are appropriate before proceeding to the next sequence of lemmas. If we define $\mathscr{S}_{\lambda} f(a)$ by (2.18), then by (2.14) and (3.11)

$$
\mathscr{S}_{\lambda} f(a)=\int_{\bar{N}} K(v: a) \tau_{\lambda}\left(\kappa\left(\delta_{a} v\right) w\right)^{-1} W^{w} f(v) d v
$$

or

$$
\mathscr{S}_{\lambda} f(a)=\int_{\bar{N}} e^{\rho H(v)} K(v: a) \tau_{\lambda}\left(\pi\left(\delta_{a} v\right) w\right)^{-1} f(\kappa(v) w) d v
$$

We have now shown that the integrand converges pointwise, except for $v=1$, to $\left(\sigma_{\lambda}(v w)^{-1} /|v|\right) W^{w} f(v)$, but since $|v|^{-1}$ just fails to be integrable, the dominated convergence theorem is not applicable. Instead, we obtain more precise information about the rate at which $\kappa\left(\delta_{a} v\right) w$ approaches $m(v w)$ in the case, essentially, of $S U(2,1)$ to which the general solution can be reduced. Positive constants that appear in these lemmas depend in an essential way only on the subscripted objects and may change from line to line. Let $B$ denote the Killing form on $\mathfrak{g}$ and let $B_{\theta}$ denote the positive definite norm on $g$ given by $B_{\theta}=B \circ(1 \times-\theta)$. The associated norm on $\mathrm{g}$ will be denoted by $\|\cdot\|$.

Lemma 3.5. Suppose $Y$ and $Z$ are nonzero elements of $\mathrm{g}^{-\alpha}$ and $\mathrm{g}^{-2 \alpha}$ respectively. Then

$$
\begin{gathered}
{[Z, \theta Z] \in \mathfrak{a},} \\
{[Y,[Y, \theta Z]] \text { is a nonzero element of } \mathrm{m},}
\end{gathered}
$$

for two positive constants $c_{0}$ and $c_{1}$.

Proof. The first statement is obvious, the second can be found in [7, Lemma 1.8], and the third is an immediate computation. For (3.20), observe that

$$
\begin{aligned}
B_{\theta}\left((\operatorname{ad} Y)^{4} \theta Z, Z\right) & =-B([Y,[Y, \theta Z]],[Y,[Y, \theta Z]]) \\
& =\|[Y,[Y, \theta Z]]\|^{2},
\end{aligned}
$$

since by (3.18) $[Y,[Y, \theta Z]]$ belongs to $\mathfrak{f}$ and so is $\theta$-invariant. 
Let $d\left(k, k^{\prime}\right)$ denote a translation invariant metric on $K$. For $X$ in $\mathfrak{n}$ let $k_{X}=\exp (X+\theta X)$.

LEMMA 3.6. There exists a neighborhood $I$ of 0 in $\mathfrak{n}$ and a positive number $\varepsilon_{0}$ such that

$$
d\left(k_{X}, 1\right) \leq C_{I} e^{-\varepsilon_{0} \rho \log a\left(k_{X^{w}}\right)} \quad(X \in I) .
$$

Proof. We give the proof for the case where $\Phi^{+}=\{\alpha, 2 \alpha\}$, it being the more difficult. The modifications necessary when $\Phi^{+}=\{\alpha\}$ are evident in the proof. For $X$ in $\mathfrak{n}$ write $\theta X=Y+Z$ with $Y$ in $\mathbf{R} \cdot X_{-\alpha}$ and $Z$ in $\mathbf{R} \cdot X_{-2 \alpha}$ where $X_{-\alpha}$ and $X_{-2 \alpha}$ are nonzero vectors in $\mathrm{g}^{-\alpha}$ and $\mathfrak{g}^{-2 \alpha}$ respectively. Let $\mathfrak{g}_{X}$ be the Lie subalgebra of $g$ generated by $X_{-\alpha}, X_{-2 \alpha}$, $\theta X_{-\alpha}$, and $\theta X_{-2 \alpha}$ and let $G_{X}$ be the analytic subgroup of $G$ corresponding to $\mathfrak{g}_{X}$. Then $\mathfrak{g}_{X}$ is isomorphic to $\mathfrak{g} \mathfrak{u}(2,1)[7$, p. 54] and direct computation shows that the analogue $\rho_{X}$ of $\rho$ for $g_{X}$ is given by $\rho_{X}=2 \alpha$. Let $\pi_{X}$ be the representation of $G_{X}$ constructed in the proof of Lemma 3.1 so that in fact $\pi_{X}$ acts on $\left(\mathfrak{g}_{X}, B_{\theta}\right)$ with $\pi_{X}(X+\theta X)=\operatorname{ad}_{\mathfrak{g}_{X}}(X+\theta X)$. We carry over from Lemma 3.1 the notation for weight vectors and projections. In a neighborhood $I$ of 0 in $\mathfrak{n}$ we have

$$
\begin{aligned}
\| E_{-2 \alpha}\left(\sum_{n=0}^{4} \frac{1}{n !} \pi_{X}(\right. & \left.X+\theta X)^{n}\right) \phi_{-2 \alpha} \|^{2} \\
& \leq c_{I}\left\|E_{-2 \alpha} \exp \pi_{X}(X+\theta X) \phi_{2 \alpha}\right\|^{2} .
\end{aligned}
$$

But the left side of (3.22) is

$$
\begin{aligned}
\|\left[\frac{1}{2}(\operatorname{ad} Z)^{2}+\frac{1}{6} \operatorname{ad} Y \operatorname{ad} Z \operatorname{ad} Y\right. & +\frac{1}{6}(\operatorname{ad} Y)^{2} \operatorname{ad} Z \\
& \left.+\frac{1}{6} \operatorname{ad} Z(\operatorname{ad} Y)^{2}+\frac{1}{24}(\operatorname{ad} Y)^{4}\right] \phi_{2 \alpha} \|^{2}
\end{aligned}
$$

and since $[Y, Z]=0$, hence $\operatorname{ad} Z(\operatorname{ad} Y)^{2}=\operatorname{ad} Y \operatorname{ad} Z \operatorname{ad} Y=(\operatorname{ad} Y)^{2} \operatorname{ad} Z$, (3.22) for $X$ in $I$ simplifies to

$$
\left\|\left[\frac{1}{2}(\operatorname{ad} Z)^{2}+\frac{1}{2}(\operatorname{ad} Y)^{2} \operatorname{ad} Z+\frac{1}{24}(\operatorname{ad} Y)^{4}\right] \phi_{2 \alpha}\right\|^{2} \leq c_{I}\left\|E_{-2 \alpha} \pi_{X}\left(k_{X}\right) \phi_{2 \alpha}\right\|^{2} .
$$

Now, if $Z \neq 0$,

$$
\phi_{2 \alpha}=\|Z\|^{-1} \theta Z,
$$

hence by (3.17) $(\operatorname{ad} Y)^{2}(\operatorname{ad} Z) \phi_{2 \alpha}=0$, so that

$$
\left\|\frac{1}{2}(\operatorname{ad} Z)^{2} \phi_{2 \alpha}+\frac{1}{24}(\operatorname{ad} Y)^{4} \phi_{2 \alpha}\right\|^{2} \leq c_{I}\left\|E_{-2 \alpha} \pi_{X}\left(k_{X}\right) \phi_{2 \alpha}\right\|^{2} .
$$

Substituting in (3.23) and using (3.19) and (3.20) we get

$$
2|\alpha|^{2}\|Z\|^{2}+\frac{1}{24} c_{0}\|Y\|^{4} \leq c_{I}\left\|E_{-2 \alpha} \pi_{X}\left(k_{X}\right) \phi_{2 \alpha}\right\| .
$$


By shrinking the neighborhood $I$ If necessary so that if $X \in I$

$$
d\left(k_{X}, 1\right) \leq c_{I}\|Y+Z\| \leq c_{I}\left(2|\alpha|^{2}\|Z\|^{2}+\frac{1}{24} c_{0}\|Y\|^{4}\right)^{1 / 4} .
$$

we have in view of (3.9)

$$
d\left(k_{X}, 1\right) \leq c_{I}\left(e^{\rho_{X} \log a\left(k_{X}^{-1} w\right)}\right)^{1 / 4}=c_{I} e^{-(p+2 q)^{-1} \rho \log a\left(k_{X}^{-1} w\right)} .
$$

Since $d\left(k_{X}^{-1}, 1\right)=d\left(k_{X}, 1\right)$, chosing $I$ to be symmetric we get

$$
d\left(k_{X}, 1\right) \leq c_{I} e^{-\varepsilon_{0} \rho \log a\left(k_{X} w\right)}
$$

with $\varepsilon_{0}=(p+2 q)^{-1}$.

Proposition 3.7. The operator valued map of $\bar{N}$ into $\operatorname{End}_{\mathbf{C}}\left(V_{\lambda}\right)$ defined by $v \rightarrow e^{\rho H(v)}\left[\tau_{\lambda}(\kappa(v) w)^{-1}-\sigma_{\lambda}(v w)^{-1}\right]$ is integrable.

Proof. Because $m(v w)=m(\kappa(v) w)$, we have the Lipschitz inequality

$$
\left\|\tau_{\lambda}(\kappa(v) w)^{-1}-\sigma_{\lambda}(v w)^{-1}\right\| \leq c d(\kappa(v) w, m(\kappa(v) w)) .
$$

Thus, in view of (2.6) it suffices to show that for some $\varepsilon>0$

$$
d(\kappa(v) w, m(\kappa(v) w)) \leq c e^{\varepsilon \rho H(v)}
$$

for $|v|$ sufficiently large. But by Proposition 3.4, for $|v|$ sufficiently large $k=m(\kappa(v) w)^{-1} \kappa(v) w$ is sufficiently close to 1 so that the neighborhood $I$ in Lemma 3.6 may be used as a chart via $k_{X}=\exp (X+\theta X)$. Thus, by (3.21)

$$
d(\kappa(v) w, m(\kappa(v) w)) \leq c_{I} e^{-\varepsilon_{0} \rho \log a\left(m(\kappa(v) w)^{-1} \kappa(v) w^{2}\right)} .
$$

Since $a\left(m(\kappa(v) w)^{-1} \kappa(v) w^{2}\right)=a(\kappa(v))=-H(v)$, (3.25) follows.

We may now now use Proposition 3.7 to deal with the singularity of $|v|^{-1}$ at $v=1$. To do so we will construct a modification of a partition of unity found in [10]. Following [10, p. 521, 523] we identify $\bar{N}$ and $\theta n$ and transfer the norm $\|\cdot\|$ to $\bar{N}$. The norm on $V_{\lambda}$ will be denoted by $\|\cdot\|_{V_{\lambda}}$. Fix any positive number $R_{0}$ (with the intention of doing a Taylor expansion in $\left.\left\{\|v\|<R_{0}\right\}\right)$. Let $\varphi(s)$ be a nonincreasing element of $C_{0}^{\infty}([0, \infty),[0,1])$ that is equal to 1 for $0 \leq s \leq d$ and to 0 for $b \leq s<\infty$ where $0<d<b$ and $b$ is chosen so that $\{|v|<b\} \subset\left\{\|v\|<R_{0}\right\}$ (cf. [10, p. 529]). Define $\psi_{1}(k)$ by

(3.26) $\quad \psi_{1}(k)= \begin{cases}\varphi(|v|) & \text { if } k=m \kappa(v) w \text { for some } m \in M, v \in \bar{N} \\ 0 & \text { otherwise. }\end{cases}$ 
LEMMA 3.8. The function $\psi_{1}$ defined on $K$ by (3.26) is a well-defined, left $M$-invariant, smooth separation of the two closed disjoint subsets $M$ and $M w$ of $K$, and does not depend on the function $f$.

Proof. That $\psi_{1}$ is well defined and smooth follows from the GelfandNaimark decomposition. It is clear that $\psi_{1}$ is left $M$-invariant and $\left.\psi_{1}\right|_{M w} \equiv 1$. The existence of an element $m$ in $M$ for which $\psi_{1}(m) \neq 0$ would imply the existence of an element $v$ in $\bar{N}$ for which $\kappa(v)$ belongs to $M w$, contradicting the disjointness of $\bar{N}$ and $M A N w$. Thus $\left.\psi_{1}\right|_{M} \equiv 0$.

Let $\psi_{2}=1-\psi_{1}, \varphi_{1}=\varphi$, and $\varphi_{2}=1-\varphi_{1}$. Thus $\psi_{i} f$ is in $C^{\infty}\left(K, \sigma_{\lambda}\right)$ $(i=1,2)$ and $\mathscr{S}_{\lambda} f=\mathscr{S}_{\lambda}\left(\psi_{1} f\right)+\mathscr{S}_{\lambda}\left(\psi_{2} f\right)$. Let $Y f(v)=W^{w} f(v)-$ $f(w) e^{\rho H(v)}$ and let $Z f(v)=f(\kappa(v) w)-f(w)$, that is, $Y f(v)=$ $e^{\rho H(v)} Z f(v)$. Then

$$
\begin{aligned}
\mathscr{S}_{\lambda} f(a)= & \sum_{i=1}^{2} \int_{\bar{N}} \varphi_{i}(|v|) K(v: a) \tau_{\lambda}\left(\kappa\left(\delta_{a} v\right) w\right)^{-1} Y f(v) d v \\
& +\int_{\bar{N}} K(v: a) \tau_{\lambda}\left(\kappa\left(\delta_{a} v\right) w\right)^{-1} f(w) e^{\rho H(v)} d v
\end{aligned}
$$

We will deal with the integrals in (3.27) in the order in which they are written. With $\nu$ an integer to be determined by Lemma 3.9, the function $Z f(v)$ has a Taylor expansion in $\left\{\|v\|<R_{0}\right\}$ of the form (cf. [10, p. 523])

$$
Z f(v)=\sum_{j=1}^{2 \nu} f_{j}(v)+R_{\nu}(v)
$$

where $f_{j}(v)$ is $\alpha$-homogeneous of degree $j$ and

$$
\left\|R_{\nu}(v)\right\|_{V_{\lambda}} \leq c\|v\|^{\nu+1} \text {. }
$$

LEMMA 3.9. For $\nu$ sufficiently large

$$
\lim _{a \rightarrow \infty} \int_{\bar{N}} \varphi(|v|) K(v: a) \tau_{\lambda}\left(\kappa\left(\delta_{a} v\right) w\right)^{-1} e^{\rho H(v)} R_{\nu}(v) d v
$$

exists and equals

$$
\int_{\bar{N}} \varphi(|v|) \frac{\sigma_{\lambda}(v w)^{-1}}{|v|} e^{\rho H(v)} R_{\nu}(v) d v .
$$

Proof. By (3.12), (3.13), and Proposition 3.4, the result will follow from the dominated convergence theorem if we show that $e^{\rho H(v)}|v|^{-1}\|v\|^{\nu+1} \chi_{\{|v|<b\}}(v)$ is integrable for $\nu$ sufficiently large. This follows from [10, p. $529(10.2)]$. 
LEMMA 3.10. For each $j=1, \ldots, 2 \nu$

$$
\lim _{a \rightarrow \infty} \int_{\bar{N}} \varphi(|v|) K(v: a) \tau_{\lambda}\left(\kappa\left(\delta_{a} v\right) w\right)^{-1} e^{\rho H(v)} f_{j}(v) d v
$$

exists and equals

$$
\int_{\bar{N}} \varphi(|v|) \frac{\sigma_{\lambda}(v w)^{-1}}{|v|} e^{\rho H(v)} f_{j}(v) d v
$$

Proof. Here it suffices to exhibit the integrability of the dominating function

$$
G_{j}(v)=|v|^{-1}\left\|f_{j}(v)\right\|_{V_{\lambda}} \chi_{\{|v|<b\}}(v) .
$$

Now

$$
h_{j}(v)=|v|^{-j /(p+2 q)}\left\|f_{j}(v)\right\|_{V_{\lambda}}
$$

is $\alpha$-homogeneous of degree 0. By [10, Proposition 3] there exists a real number $e\left(h_{j}\right)$ for which

$$
\int_{\bar{N}} G_{j}(v) d v=e\left(h_{j}\right) \int_{0}^{\infty}\left(\frac{1}{r}\right)^{1-j /(p+2 q)} \varphi(r) d r .
$$

The right hand side is clearly finite for any $j>0$.

Corollary 3.11. For $f$ in $C^{\infty}\left(K, \sigma_{\lambda}\right)$,

$$
\lim _{a \rightarrow \infty} \int_{\bar{N}} \varphi(|v|) K(v: a) \tau_{\lambda}\left(\kappa\left(\delta_{a} v\right) w\right)^{-1} Y f(v) d v
$$

exists and equals

$$
\int_{\bar{N}} \varphi(|v|) \frac{\sigma_{\lambda}(v w)^{-1}}{|v|} Y f(v) d v
$$

LeMma 3.12. For $f$ in $C^{\infty}\left(K, \sigma_{\lambda}\right)$,

$$
\lim _{a \rightarrow \infty} \int_{\bar{N}} \varphi_{2}(|v|) K(v: a) \tau_{\lambda}\left(\kappa\left(\delta_{a} v\right) w\right)^{-1} Y f(v) d v
$$

exists and equals

$$
\int_{\bar{N}} \varphi_{2}(|v|) \frac{\sigma_{\lambda}(v w)^{-1}}{|v|} Y f(v) d v
$$




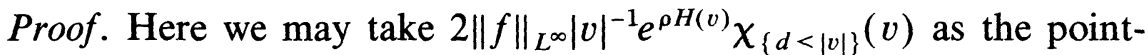
wise dominating function and its integrability follows from (2.6) and (3.6).

Lemma 3.12 together with Corollary 3.11 show that

$$
\lim _{a \rightarrow \infty} \mathscr{S}_{\lambda}(Y f)(a)=\int_{\bar{N}} \frac{\sigma_{\lambda}(v w)^{-1}}{|v|} Y f(v) d v .
$$

Only the last integral in (3.27) remains. As a first step in this consideration we write

$$
\begin{aligned}
& \int_{\bar{N}} K(v: a) \tau_{\lambda}\left(\kappa\left(\delta_{a} v\right) w\right)^{-1} f(w) e^{\rho H(v)} d v \\
& \quad=\int_{\bar{N}} \xi_{a}(v) f(w) e^{\rho H(v)} d v+\int_{\bar{N}} K(v: a) \sigma_{\lambda}(v w)^{-1} f(w) e^{\rho H(v)} d v
\end{aligned}
$$

where $\xi_{a}: \bar{N} \rightarrow$ End $V_{\lambda}$ is defined by

$$
\xi_{a}(v)=K(v: a)\left[\tau_{\lambda}\left(\kappa\left(\delta_{a} v\right) w\right)^{-1}-\sigma_{\lambda}(v w)^{-1}\right] .
$$

Note that by Proposition $3.7 \xi_{1}(v)=e^{\rho H(v)}\left[\tau_{\lambda}(\kappa(v) w)^{-1}-\sigma_{\lambda}(v w)^{-1}\right]$ is integrable. Furthermore, by the homogeneity property (3.5) and by (3.14),

$$
\xi_{a}(v) d v=\xi_{1}\left(\delta_{a} v\right) d\left(\delta_{a} v\right) .
$$

Thus, if we let $\Xi$ be the element of $\operatorname{End}\left(V_{\lambda}\right)$ given by

$$
\Xi=\int_{\bar{N}} \xi_{1}(v) d v
$$

a standard approximation to the identity argument gives

$$
\lim _{a \rightarrow \infty} \int_{\bar{N}} \xi_{a}(v) f(w) e^{\rho H(v)} d v=\Xi f(w)
$$

and so we may turn our attention to establishing the limit of the last term in (3.31). We introduce the truncated kernel

$$
K^{\prime}(v: a)= \begin{cases}|v|^{-1} & \text { if } e^{-2 \rho \log a} \leq|v| \\ 0 & \text { otherwise }\end{cases}
$$

and we let

$$
\theta_{a}(v)\left[K(v: a)-K^{\prime}(v: a)\right] \sigma_{\lambda}(v w)^{-1} .
$$

It is a simple matter using (3.6) to verify that $\theta_{1}(v)$ belongs to $L^{1}\left(\widetilde{N}, \operatorname{End}\left(H_{\lambda}\right)\right)$. Furthermore, $\theta_{a}(v) d v=\theta_{1}\left(\delta_{a} v\right) d\left(\delta_{a} v\right)$ by (3.2) and 
(3.4). If we let $\vartheta$ be the element of $\operatorname{End}\left(H_{\lambda}\right)$ given by

$$
\vartheta=\int_{\bar{N}} \theta_{1}(v) d v
$$

then an approximation to the identity argument gives

$$
\lim _{a \rightarrow \infty} \int_{\bar{N}} \theta_{a}(v) f(w) e^{\rho H(v)} d v=\vartheta f(w)
$$

LEMMA 3.13.

$$
\lim _{a \rightarrow \infty} \int_{\bar{N}} K(v: a) \sigma_{\lambda}(v w)^{-1} f(w) e^{\rho H(v)} d v
$$

exists and equals

$$
\begin{aligned}
\int_{|v| \leq 1} & \frac{e^{\rho H(v)}-1}{|v|} \sigma_{\lambda}(v w)^{-1} f(w) d v \\
& +\int_{1<|v|} \frac{e^{\rho H(v)}}{|v|} \sigma_{\lambda}(v w)^{-1} f(w) d v+\vartheta f(w)
\end{aligned}
$$

Proof.

$$
\begin{aligned}
\int_{\bar{N}} K^{\prime}(v: a) \sigma_{\lambda}(v w)^{-1} f(w) e^{\rho H(v)} d v \\
=\int_{e^{-2 \rho \log a} \leq|v|} \frac{e^{\rho H(v)}}{|v|} \sigma_{\lambda}(v w)^{-1} f(w) d v \\
=\int_{e^{-2 \rho \log a} \leq|v| \leq 1} \frac{e^{\rho H(v)}}{|v|} \sigma_{\lambda}(v w)^{-1} f(w) d v \\
\quad+\int_{1<|v|} \frac{e^{\rho H(v)}}{|v|} \sigma_{\lambda}(v w)^{-1} f(w) d v
\end{aligned}
$$

Now

$$
\int_{e^{-2 p \log a} \leq|v| \leq 1} \frac{\sigma_{\lambda}(v w)^{-1}}{|v|} f(w) d v=0
$$

for every $a$ by [10, Proposition 20] so we may subtract it from the first term on the right hand side above. Since $\left(e^{\rho H(v)}-1\right)|v|^{-1}$ is continuous on the compact set $\{|v| \leq 1\}$, the lemma follows by decomposing $K(v: a)$ as $K(v: a)=\theta_{a}(v)+K^{\prime}(v: a)$ and using (3.38). 
We combine the results of (3.27), Corollary 3.11, Lemma 3.12, (3.31), (3.34), and Lemma 3.13 as

TheOREM 3.14. Let $f$ belong to $C^{\infty}\left(K, \sigma_{\lambda}\right)$ and let $\mathscr{S}_{\lambda}$, $\Xi$ and $\vartheta$ be the operators defined by (2.18), (3.33), and (3.37) respectively. Then

$$
\begin{aligned}
\lim _{a \rightarrow \infty} \mathscr{S}_{\lambda} f(a)= & \Xi f(w)+\vartheta f(w) \\
& +\int_{\bar{N}} \frac{\sigma_{\lambda}(v w)^{-1}}{|v|}\left[W^{w} f(v)-e^{\rho H(v)} f(w)\right] d v \\
& +\int_{|v| \leq 1} \frac{\sigma_{\lambda}(v w)^{-1}}{|v|}\left[e^{\rho H(v)} f(w)-f(w)\right] d v \\
& +\int_{1<|v|} \frac{\sigma_{\lambda}(v w)^{-1}}{|v|} e^{\rho H(v)} f(w) d v .
\end{aligned}
$$

We can write this result in a more convenient form through the use of principal value integrals. Thus, for $F$ in $L^{2}\left(\bar{N}, H_{\lambda}\right)$ we make the following interpretation of the singular inegral with kernel $|v|^{-1} \sigma_{\lambda}(v w)^{-1}$ :

$$
\int_{\bar{N}}|v|^{-1} \sigma_{\lambda}(v w)^{-1} F(v) d v=\lim _{\varepsilon \rightarrow 0} \int_{\varepsilon<|v|}|v|^{-1} \sigma_{\lambda}(v w)^{-1} F(v) d v .
$$

Then, by using the mean value zero property (1.5) of $|v|^{-1} \sigma_{\lambda}(v w)^{-1}$ on spherical shells, we can rewrite the conclusion of Theorem 3.14 as

(3.39) $\lim _{a \rightarrow \infty} \mathscr{S}_{\lambda} f(a)=\Xi f(w)+\vartheta f(w)+\int_{\bar{N}} \frac{\sigma_{\lambda}(v w)^{-1}}{|v|} W^{w} f(v) d v$.

Furthermore, the same device shows that

$$
\int_{\bar{N}} e^{\rho H(v)} \tau_{\lambda}(\kappa(v) w)^{-1} f(w) d v
$$

has meaning as a principal value integral and in fact

$$
\lim _{V \rightarrow \infty} \int_{|v|<V} e^{\rho H(v)} \tau_{\lambda}(\kappa(v) w)^{-1} f(w) d v=(\Xi+\vartheta) f(w)
$$

Thus,

$$
\begin{aligned}
\lim _{a \rightarrow \infty} \mathscr{S}_{\lambda} f(a)= & \int_{\bar{N}} e^{\rho H(v)} \tau_{\lambda}(\kappa(v) w)^{-1} f(w) d v \\
& +\int_{\bar{N}} \frac{\sigma_{\lambda}(v w)^{-1}}{|v|} W^{w} f(v) d v
\end{aligned}
$$


Let $E_{\lambda}$ be the projection of $V_{\lambda}$ onto $H_{\lambda}$. Observe that the last term in (3.40) already has values in $H_{\lambda}$ and is not affected by the projection $E_{\lambda}$.

LEMMA 3.15. There exists a constant $a_{\lambda}$ such that (3.41) $\lim _{a \rightarrow \infty} E_{\lambda} \mathscr{S}_{\lambda} f(a)=a_{\lambda} f(w)+\int_{\bar{N}}|v|^{-1} \sigma_{\lambda}(v w)^{-1} W^{w} f(v) d v$.

Proof. That

$$
E_{\lambda} \int_{\bar{N}} e^{\rho H(v)} \tau_{\lambda}(\kappa(v) w)^{-1} d v=a_{\lambda} I
$$

is a consequence of Schur's lemma. Indeed, for $m$ in $M$, we have $w m=m w, m^{-1} \kappa(v) m=\kappa\left(m^{-1} v m\right)$, and $d\left(m v m^{-1}\right)=d v$. Hence, $\sigma_{\lambda}(m)$ and

$$
E_{\lambda} \int_{\bar{N}} e^{\rho H(v)} \tau_{\lambda}(\kappa(v) w)^{-1} d v
$$

commute.

To each element $F$ in the image of $S_{\lambda}$ in $C^{\infty}\left(G, \tau_{\lambda}\right)$ we now associate a function $\mathscr{L F}$ on $K$ with values in $H_{\lambda}$ as follows:

$$
\mathscr{L} F(k)=\lim _{a \rightarrow \infty} E_{\lambda} \mathscr{S}_{\lambda}\left({ }^{w^{-1} k} f\right)(a) \quad\left(F=S_{\lambda} f ; f \in C^{\infty}\left(K, \sigma_{\lambda}\right)\right) .
$$

Using the form of $S_{\lambda}$ given in (1.3) we have

$$
\mathscr{L} F(k)=\lim _{a \rightarrow \infty} e^{\rho \log a} E_{\lambda} \int_{K} \tau_{\lambda}(l)^{-1} f\left(l a w^{-1} k\right) d l,
$$

from which it follows that

$$
\mathscr{L} F(m k)=\sigma_{\lambda}(m) \mathscr{L} F(k) .
$$

We extend the definition of $\mathscr{L} F$ to $G$ by

$$
\mathscr{L} F(g)=e^{\rho H(g)} \mathscr{L} F(\kappa(g)) .
$$

THEOREM 3.16. The boundary value map $\mathscr{L}$ defined by (3.42) maps $S_{\lambda}\left(C^{\infty}\left(K, \sigma_{\lambda}\right)\right)$ into $L^{2}\left(K, \sigma_{\lambda}\right)$ in a G-equivariant manner. Furthermore, the intertwining operator that is the composite

$$
\mathscr{U}\left(\sigma_{\lambda}: \rho\right) \stackrel{W^{-1}}{\rightarrow} U\left(\sigma_{\lambda}: \rho\right) \stackrel{\mathscr{L} \circ S_{\lambda}}{\rightarrow} U\left(\sigma_{\lambda}: \rho\right) \stackrel{W}{\rightarrow} \mathscr{U}\left(\sigma_{\lambda}: \rho\right)
$$

is the projection $a_{\lambda} I+\int_{\bar{N}}|v|^{-1} \sigma_{\lambda}(v w)^{-1} F(v \cdot) d v$, i.e.

$$
W \mathscr{L}\left(S_{\lambda} W^{-1} F\right)(u)=a_{\lambda} F(u)+\int_{\bar{N}}|v|^{-1} \sigma_{\lambda}(v w)^{-1} F(v u) d v
$$

for a smooth element $F$ in $L^{2}\left(\bar{N}, H_{\lambda}\right)$. 
Proof. Since $S_{\lambda}$ is $G$-equivariant, to establish the $G$-equivariance of $\mathscr{L}$ it must be shown that

$$
\mathscr{L}\left(S_{\lambda} U(g) f\right)(x)=\mathscr{L}\left(S_{\lambda} f\right)(x g) \quad(x, g \in G) .
$$

It suffices to prove (3.45) when $x=1$. For $g$ in $K$ this follows form the definition. For $g=a_{0} \in A$ we have by (3.43)

$$
\begin{aligned}
\mathscr{L}\left(S_{\lambda} U\left(a_{0}\right) f\right)(1) & =\lim _{a \rightarrow \infty} e^{\rho \log a} E_{\lambda} \int_{K} \tau_{\lambda}(l)^{-1} f\left(\operatorname{law}^{-1} a_{0}\right) d l \\
& =e^{\rho \log a_{0}} \lim _{a \rightarrow \infty} e^{\rho \log a a_{0}^{-1}} E_{\lambda} \int_{K} \tau_{\lambda}(l)^{-1} f\left(l a a_{0}^{-1} w^{-1}\right) d l \\
& =e^{\rho \log a_{0}} \mathscr{L}\left(S_{\lambda} f\right)(1)=\mathscr{L}\left(S_{\lambda} f\right)\left(a_{0}\right) .
\end{aligned}
$$

It follows from the Cartain decomposition $G=K A K$ that (3.46) holds for every $g$ when $x=1$. Finally, from the explicit limit formula given in Theorem 3.14, it is clear that $\mathscr{L} F(k)$ is continuous; hence, $\mathscr{L} F$ belongs to $L^{2}\left(K, \sigma_{\lambda}\right)$. This proves the first part.

Now let $F$ be a smooth element of $L^{2}\left(\bar{N}, H_{\lambda}\right)$. Then

$$
\left(W^{-1} F\right)(k)=e^{\rho \log a(k)} \sigma_{\lambda}(m(k)) F(\bar{n}(k)) .
$$

By (3.41) and (3.42) we get

$$
\mathscr{L}\left(S_{\lambda} W^{-1} F\right)(k)=a_{\lambda} W^{-1} F(k)+\int_{\bar{N}}|v|^{-1} \sigma_{\lambda}(v w)^{-1} W\left({ }^{k} W^{-1} F\right)(v) d v .
$$

Since $W, W^{-1}$, and $\mathscr{L} \circ S_{\lambda}$ are equivariant, we have for $u$ in $\bar{N}$

$$
\begin{aligned}
W\left(\mathscr{L}\left(S_{\lambda} W^{-1} F\right)\right)(u) & =W\left(\mathscr{L}\left(S_{\lambda} W^{-1} \mathscr{U}(u) F\right)\right)(1) \\
& =e^{\rho H(1)} \mathscr{L}\left(S_{\lambda} W^{-1} \mathscr{U}(u) F\right)(1) \\
& =a_{\lambda} F(u)+\int_{\bar{N}}|v|^{-1} \sigma_{\lambda}(v w)^{-1} F(v u) d v .
\end{aligned}
$$

\section{REFERENCES}

[1] V. Bargmann, Irreducible unitary representations of the Lorentz group, Ann. of Math., (2) (1947), 568-640.

[2] Brian E. Blank, Embedding limits of discrete series of semisimple Lie groups, Canadian Math. Conf. Proc., 1 (1981), 55-64, American Mathematical Society, Providence, R. I., 1981.

[3] Harish-Chandra, Spherical functions on a semisimple Lie group I, Amer. J. Math., 80 (1958), 241-310.

[4] _ Discrete series for semisimple Lie groups I, Acta Math., 113 (1965), 241-318. 
[5] _ Discrete series for semisimple Lie groups II, Acta Math., 116 (1966), 1-111.

[6] S. Helgason, Differential Geometry and Symmetric Spaces, Pure and Applied Math. vol. 12, Academic Press, N. Y., 1962.

[7] _ A duality for symmetric spaces with applications to group representations, Advances in Math., 5 (1970), 1-154.

[8] A. W. Knapp, Weyl group of a cuspidal parabolic, Ann. Scient. Ec. Norm. Sup., (4) 8 (1975), 275-294.

[9] A. W. Knapp and K. Okamoto, Limits of holomorphic discrete series, J. Funct. Anal., 9 (1972), 375-409.

[10] A. W. Knapp and E. M. Stein, Intertwining operators for semisimple groups, Ann. of Math., (2) 93 (1971), 489-578.

[11] A. W. Knapp and N. R. Wallach, Szegö kernels associated with discrete series, Invent. Math., 34 (1976), 163-200.

[12] H. Midorikawa, On certain irreducible representations for the real rank one classical groups, J. Faculty Sci. Univ. of Tokyo, 21 (1974), 435-459.

[13] W. Schmid, On the realization of the discrete series of a semisimple Lie group, Rice Univ. Studies, 56 (1970), 99-108.

[14] Some properties of square integrable representations of semisimple Lie groups, Ann. of Math., (2) 102 (1975), 535-564.

Received November 27, 1984.

WASHINGTON UNIVERSITY

ST. LOUIS, MO 63130 


\section{PACIFIC JOURNAL OF MATHEMATICS EDITORS}

\author{
V. S. VARAdarajan (Managing Editor) \\ University of California \\ Los Angeles, CA 90024 \\ Hebert Clemens \\ University of Utah \\ Salt Lake City, UT 84112 \\ Charles R. DePrima \\ California Institute of Technology \\ Pasadena, CA 91125
}

R. FINN

Stanford University

Stanford, CA 94305

HeRManN FLASChKa

University of Arizona

Tucson, AZ 85721

RAMESH A. GANGOlli

University of Washington

Seattle, WA 98195

ROBION KIRBY

University of California

Berkeley, CA 94720

\author{
C. C. MOORE \\ University of California \\ Berkeley, CA 94720 \\ H. SAMELSON \\ Stanford University \\ Stanford, CA 94305 \\ HAROLD STARK \\ University of California, San Diego \\ La Jolla, CA 92093
}

\section{ASSOCIATE EDITORS}

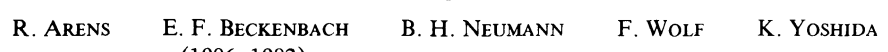

(1906-1982)

\section{SUPPORTING INSTITUTIONS}

\begin{abstract}
UNIVERSITY OF ARIZONA
UNIVERSITY OF BRITISH COLUMBIA

CALIFORNIA INSTITUTE OF TECHNOLOGY

UNIVERSITY OF CALIFORNIA

MONTANA STATE UNIVERSITY

UNIVERSITY OF NEVADA, RENO

NEW MEXICO STATE UNIVERSITY

OREGON STATE UNIVERSITY
\end{abstract}

\author{
UNIVERSITY OF OREGON \\ UNIVERSITY OF SOUTHERN CALIFORNIA \\ STANFORD UNIVERSITY \\ UNIVERSITY OF HAWAII \\ UNIVERSITY OF TOKYO \\ UNIVERSITY OF UTAH \\ WASHINGTON STATE UNIVERSITY \\ UNIVERSITY OF WASHINGTON
}

The Supporting Institutions listed above contribute to the cost of publication of this Journal, but they are not owners or publishers and have no responsibility for its content or policies.

Mathematical papers intended for publication in the Pacific Journal of Mathematics should be in typed form or offset-reproduced (not dittoed), double spaced with large margins. Please do not use built up fractions in the text of the manuscript. However, you may use them in the displayed equations. Underline Greek letters in red, German in green, and script in blue. The first paragraph must be capable of being used separately as a synopsis of the entire paper. In particular it should contain no bibliographic references. Please propose a heading for the odd numbered pages of less than 35 characters. Manuscripts, in triplicate, may be sent to any one of the editors. Please classify according to the scheme of Math. Reviews, Index to Vol. 39. Supply name and address of author to whom proofs should be sent. All other communications should be addressed to the managing editor, or Elaine Barth, University of California, Los Angeles, California 90024.

There are page-charges associated with articles appearing in the Pacific Journal of Mathematics. These charges are expected to be paid by the author's University, Government Agency or Company. If the author or authors do not have access to such Institutional support these charges are waived. Single authors will receive 50 free reprints; joint authors will receive a total of 100 free reprints. Additional copies may be obtained at cost in multiples of 50 .

The Pacific Journal of Mathematics is issued monthly as of January 1966. Regular subscription rate: $\$ 190.00$ a year (5 Vols., 10 issues). Special rate: $\$ 95.00$ a year to individual members of supporting institutions.

Subscriptions, orders for numbers issued in the last three calendar years, and changes of address should be sent to Pacific Journal of Mathematics, P.O. Box 969, Carmel Valley, CA 93924, U.S.A. Old back numbers obtainable from Kraus Periodicals Co., Route 100, Millwood, NY 10546.

The Pacific Journal of Mathematics at P.O. Box 969, Carmel Valley, CA 93924 (ISSN 0030-8730) publishes 5 volumes per year. Application to mail at Second-class postage rates is pending at Carmel Valley, California, and additional mailing offices. Postmaster: Send address changes to Pacific Journal of Mathematics, P.O. Box 969, Carmel Valley, CA 93924.

PUBLISHED BY PACIFIC JOURNAL OF MATHEMATICS, A NON-PROFIT CORPORATION

Copyright $\odot 1986$ by Pacific Journal of Mathematics 


\section{Pacific Journal of Mathematics}

\section{Vol. 122, No. 2 \\ February, 1986}

Gideon Amit and David Chillag, On a question of Feit concerning character values of finite solvable groups ......................257

Constantin Gelu Apostol and Frank Larkin Gilfeather, Isomorphisms modulo the compact operators of nest algebras ................263

Parviz Azimi and James Neil Hagler, Examples of hereditarily $l^{1}$ Banach

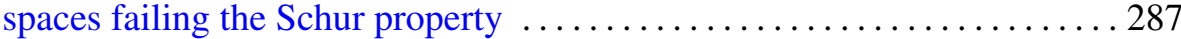

Brian Evan Blank, Boundary behavior of limits of discrete series representations of real rank one semisimple groups . . . . . . . . . . 299

Jeffrey Carroll, Some undecidability results for lattices in recursion theory

Gerald Howard Cliff and Alfred Rheinhold Weiss, Crossed product and

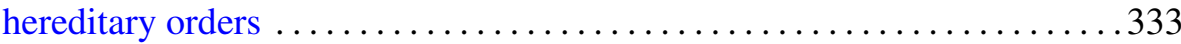

Ralph Cohen, Realizing transfer maps for ramified coverings . . . . . . . . 347

Ronald James Evans, Hermite character sums . .................. 357

C. L. Frenzen and Roderick Sue-Chuen Wong, Asymptotic expansions of the Lebesgue constants for Jacobi series . . . . . . . . . . . . . . . . 391

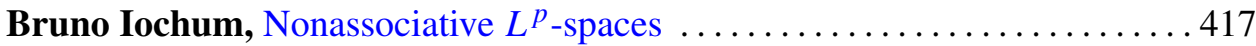

John McDonald, Unimodular approximation in function algebras ....... 435

John Robert Quine, Jr., Ramification and unintegrated value distribution . . 441

Marc Raphael, Commutants of quasisimilar subnormal operators ........ 449

Parameswaran Sankaran and Peter Zvengrowski, On stable

parallelizability of flag manifolds

Helga Schirmer, A relative Nielsen number

Barry Simon, Schrödinger semigroups on the scale of Sobolev spaces . . . . . 475

Viakalathur Shankar Sunder, Stochastic integration in Fock space

Jan de Vries, A note on the $G$-space version of Glicksberg's theorem 